\title{
Localized failure in saturated porous media
}

\author{
Jacques J.M. Desrues \\ Laboratoire 3S , CNRS - University of Grenoble, France \\ Frédéric H.J.S. Collin \\ FNRS Post-Doctoral Researcher, University of Liège, Belgique
}

Strain localisation in soils and rocks has been studied extensively for the last 20 years. A part of these studies have been devoted to the response of specimens submitted to tests in undrained situation. It was shown that shear banding can take place in both contractive and dilative specimens, with some special features due to the coupling between the granular skeleton and the pore fluid. In the paper, the relevance of the bifurcation criterion in shear band mode in the case of hydromechanical coupling is assessed by numerical study of the response of the constitutive model CLoE -the Hypoplastic model developed in L3S-Grenoble- in two kinds of numerical integration: local, i.e. at the material point level and global, i.e. in boundary value problems analyzed by finite elements.

\section{INTRODUCTION}

Strain localization in soils and rocks has been studied extensively in Laboratoire 3S, in Grenoble, for the last 20 years (Desrues and Viggiani 2004). A part of these studies have been devoted to the response of specimens submitted to tests in undrained situation (Mokni and Desrues 1999; Roger et al. 1998). It was shown, using strain field measurement methods e.g. stereophotogrammetry, that shear banding can take place in both contractive and dilative specimens, but for the latter the onset of localization is delayed until cavitation takes place in the pore-fluid. It was concluded that in dilative granular media, non-drainage can preclude localization as long as cavitation in the pore-fluid does not relax the isochoric constraint.

In the paper, the relevance of the bifurcation criterion in shear band mode in the case of hydromechanical coupling is assessed by numerical exploration of the response of the constitutive model CLoE -the Hypoplastic model developed in L3S-Grenoble (Chambon, Desrues, Charlier, and Hammad 1994a; Chambon, Caillerie, Desrues, and Crochepeyre 1999) - in two kinds of numerical integration: local -at the material point level- and global -in boundary value problems analyzed by finite elements.

\section{EXPERIMENTAL AND THEORETICAL BACKGROUND}

\subsection{Experimental evidence}

Before presenting a brief review of experimental features, it is worth recalling what we exactly mean by "drained" and "undrained" behavior. First of all, the term "behavior" is used here in a weak sense. Strictly speaking, in the context of continuum mechanics, "behavior" should be used for a continuum medium like the solid phase, or the liquid phase, but not for the composite medium made of interacting solid and fluid phases. Indeed, as discussed beneath, non-drainage can be a matter of boundary conditions, which is not intrinsic to the medium.

Drained behavior means that no pore pressure is generated in the porous medium undergoing a deformation process, because the pore fluid is able to flow with respect to the skeleton. Volumetric strain is possible. Permeable boundaries, and slow process are necessary. 
Undrained behavior means that pore pressure is generated because the pore fluid cannot flow with respect to the skeleton to accommodate the deformation process. The deformation is isochoric (no volume change). This can result from different situations : impermeable boundaries of the domain -e.g. laboratory test specimen-, very rapid loading process -e.g. earthquakes-, very low permeability -e.g. clays, argiliceous rocks.

Furthermore, in Undrained situations, it is necessary not to confuse Globally undrained and Locally undrained situations.

In Globally undrained situations, relative drainage can appear between zones of the domain, for example, between shear bands and blocks as far as localization is concerned. A concept of shear band patterns with an alternance of dilating shear bands, and contracting inter-bands zones exchanging fluid inside a specimen without global volume change was proposed and observed experimentally in one case- by Vardoulakis (Han and Vardoulakis 1991). This concept is illustrated by figure 1 .

In Locally undrained situations, no relative drainage can take place, volumetric strain is zero in every point in the domain. Pore pressure field will become heterogeneous if shear banding tends to start. Local reinforcement/weakening can be expected. But this may be vanishing with time, due to delayed relative drainage (diffusion). This process can be unsafe.

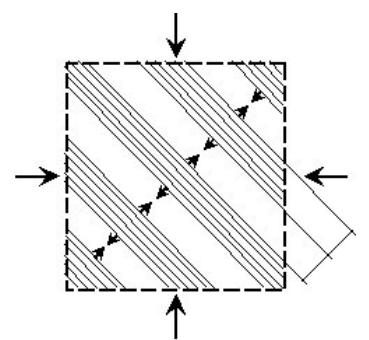

Figure 1: Alterned dilating shear bands and contracting bands in a globally undrained volume, after Han and Vardoulakis, 1991.

Strain localization in soils and granular materials. During the last 20 years, a lot of work has been devoted to strain localization in solids, both on the experimental and theoretical sides. It is well known that strain localization is associated with rupture in many solids, from metals to geomaterials, including polymers, ceramics and other solids. As for soils and granular materials, experimental studies performed by Vardoulakis and co-workers (Vardoulakis, Goldscheider, and Gudehus 1978; Vardoulakis and Graf 1985; Han and Vardoulakis 1991), Tatsuoka and coworkers e.g.(Tatsuoka, Sakamoto, Kawamura, and Fukushima 1986; Tatsuoka, Nakamura, Huang, and Tani 1990), Arthur (Arthur, Dunstan, Al-ani, and Assadi 1977; Arthur and Dunstan 1982), Finno, Viggiani et al. (Finno, Harris, Mooney, and Viggiani 1996; Finno, Harris, Mooney, and Viggiani 1997), Desrues and co-workers (Desrues and Viggiani 2004; Desrues 1984; Desrues 1990; Desrues, Lanier, and Stutz 1985; Desrues, Chambon, Mokni, and Mazerolle 1996; Mokni and Desrues 1999; Bésuelle, Desrues, and Raynaud 2000), and others (many other studies in rock) have established a number of conclusions, among which the following can be listed:

- Strain Localization in Shear Band mode can be observed in most, if not all, laboratory tests leading to rupture in geomaterials, at least at sufficiently low temperature and pressure: axisymmetric triaxial tests, plane strain biaxial tests, direct shear tests, compression-torsion hollow cylinder tests, even cubical tests with rigid boundaries.

- Complex localization patterns may be the result of specific geometrical or loading conditions. In very short specimens, shear bands can reflect several times from the rigid boundaries of the specimen, as revealed by incremental strain field monitoring using stereophotogrammetry on a specially designed biaxial apparatus (Desrues and Viggiani 2004).

- In axisymmetric tests, strain localization may remain more or less hidden inside the specimen, especially when improved test conditions are used, with reduced slenderness and refined anti-friction devices. However, Computed Tomography has made it possible to reveal that complex localization patterns can take place inside the specimen (Desrues, Chambon, Mokni, and Mazerolle 1996; Desrues 2004); these patterns are a combination of plane strain mechanisms with reflections on the top and bottom platens of the testing device.

- Well marked stress peaks in stress-strain curves can be considered as the signature of an established shear band system over the specimens. Plane strain experiments performed by 


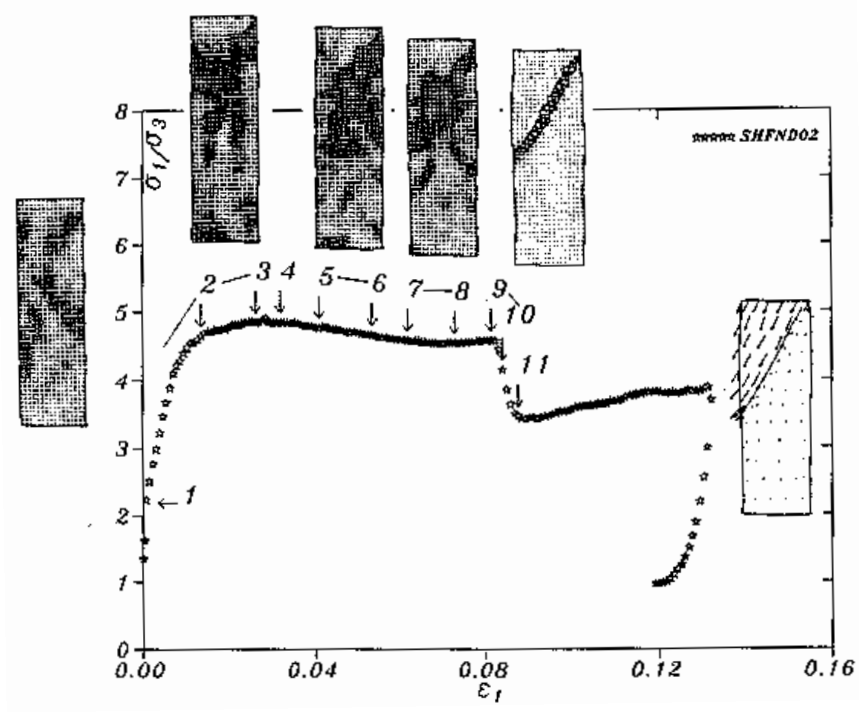

Figure 2: Strain localization in a dense Hostun sand specimen subjected to an undrained biaxial test, after Mokni and Desrues, 1999.

Desrues et al. on sand, using stereophotogrammetry to detect precisely the onset of strain localization, have shown that the onset of strain localization is always detected at or before the peak in the axial stress versus axial strain curve (Desrues 1984; Desrues 1998).

- Strain localization is observed in both drained and undrained specimens, with a number of specific features in the latter. This point is discussed in more details in the sequel.

An extended list, including more details, comments and references can be found in (Desrues and Chambon 2002).

Strain localization and drainage conditions. Only a few experimental studies have been devoted to strain localisation in undrained tests. To the authors' knowledge, besides the work performed in Grenoble by the author and co-workers (Mokni and Desrues 1999; Roger, Desrues, and Viggiani 1998), only two others studies have been conducted, both using a plane strain compression apparatus: one by Han and Vardoulakis (Han and Vardoulakis 1991) on $S^{t}$ Peter Sandstone sand (a fine sand with round grains), and another one by Finno et al. (Finno, Harris, Mooney, and Viggiani 1997) on fine masonry sand. In two papers (Vardoulakis 1996a; Vardoulakis 1996b) devoted to deformation in water-saturated sand, Vardoulakis addressed theoretically both the locally and globally undrained cases.

Evidence of strain localization in undrained tests. Plane strain undrained tests have been performed on both dense and loose Hostun sand specimens in the biaxial apparatus developed by the first author in Grenoble. Figure 2, obtained using a stereophotogrammetric survey of the specimen along the test, illustrates the fact that strain localization does occur, and occurs at a given time clearly identified on the effective stress ratio vs axial mean strain curve by a marked drop of the mobilized friction angle.

Evidence of cavitation in the pore fluid. Indeed, strain localization in undrained specimens made of dense sand proves to be possible only when cavitation occurs in the pore fluid. Figure 3 shows that, for a series of tests performed starting from different initial pore pressure (pp), higher is the initial pp, later the localization (left graph). On the right graph of the figure, it is clear that all the pore pressure curves are almost superposable by translation until each of them reaches a common plateau, at a pressure value about $-80 \mathrm{kPa}$ which is the cavitation pressure of water at ambiant temperature. The abscisse at which the cavitation pressure is reached is found to coincide with localization in every test. 

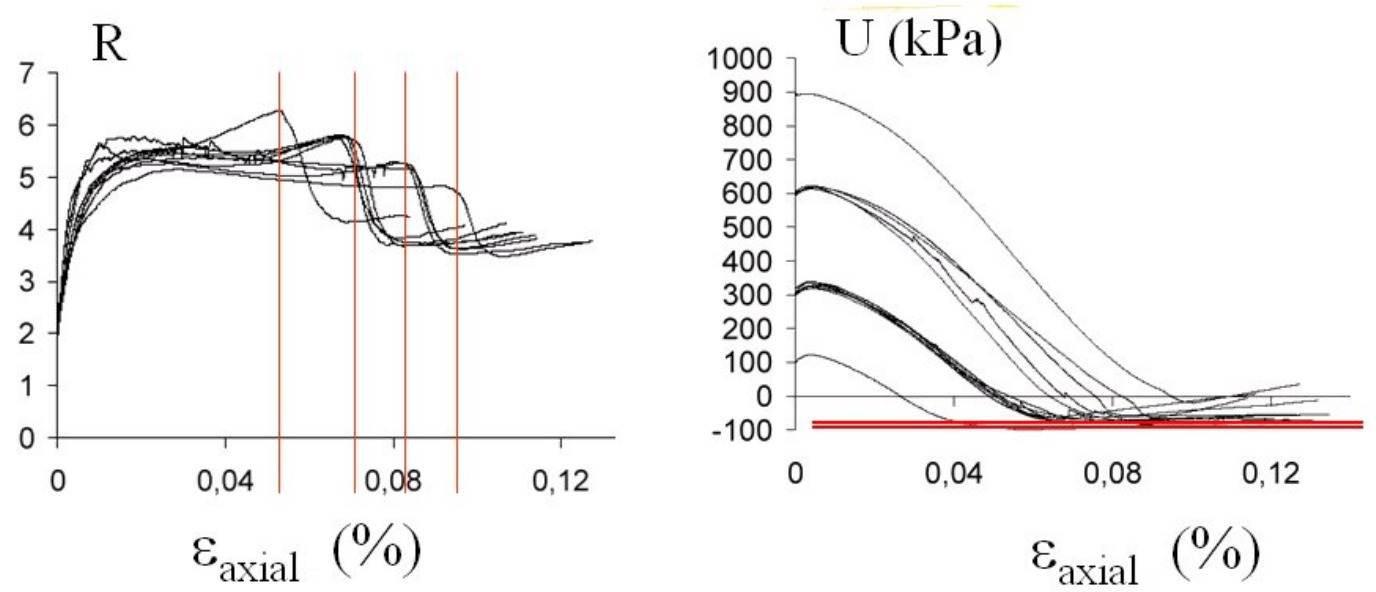

Figure 3: A set of undrained biaxial tests performed at the same initial mean effective stress, but different initial pore pressure. Localization occurs at the same time for each group performed at the same initial pore pressure (left); moreover it coincides with the time to reach cavitation pressure in the fluid (right). after Roger V., Desrues J. and Viggiani G., 1998

Stress path followed at localization. Figure 4 shows a comparison between the effective stress path followed in a drained biaxial test and an undrained one, in the $t^{\prime}, s$ space (see definitions of $t^{\prime}$ and $s$ in the figure). The drained stress path goes straight to the bifurcation line (defined from observed peak stress ratio at localization in a series of drained tests on identical specimens performed at different initial mean effective stress, not shown here). As for the undrained test, the stress path follows a line slightly below the bifurcation line, and does not localize as long as it remains on this line. However, when cavitation occurs, the stress path deviates and turns towards the bifurcation line; localization occurs when the latter is reached.

\subsection{Theoretical background}

On the theoretical side, it has been established since the years 1970 by Rice and co-workers (Rudnicki and Rice 1975; Rice 1976), following previous works by Hadamard, Hill, Mandel, that the onset of shear banding in a semi-infinite homogeneous body subjected to a homogeneous loading

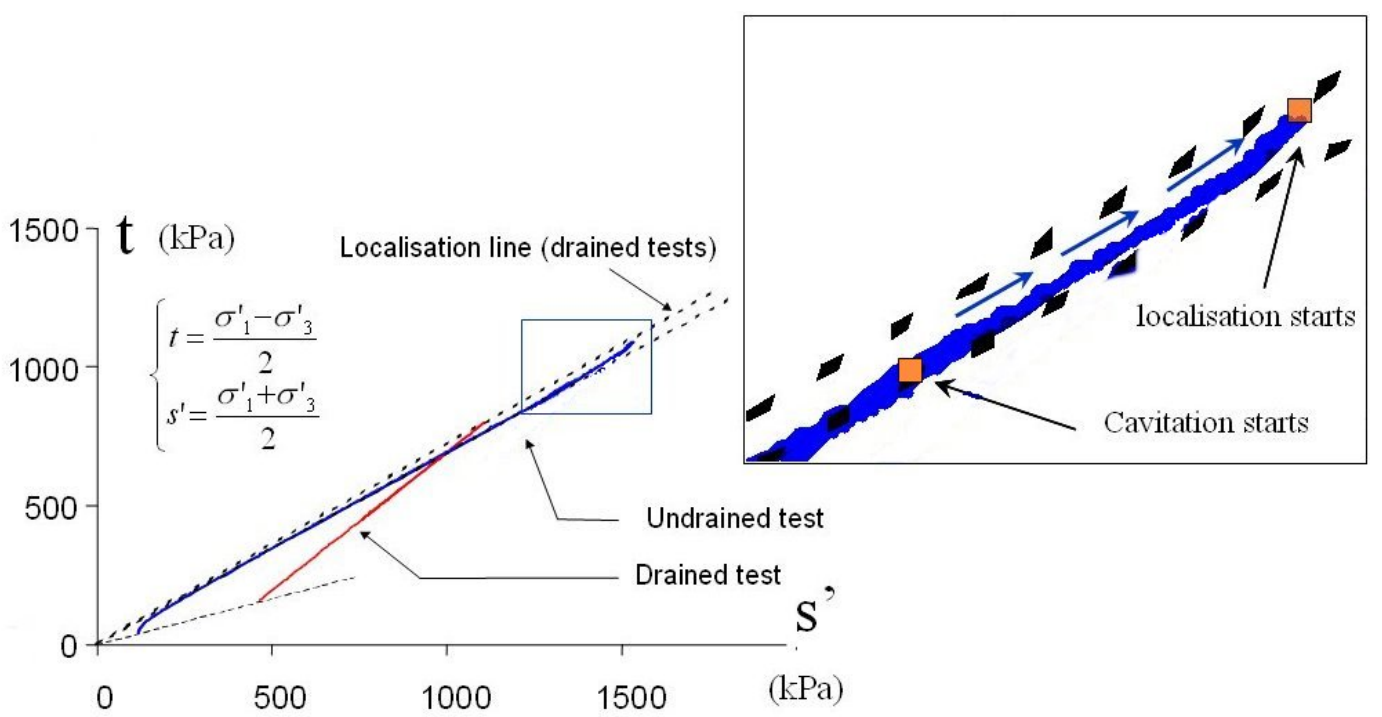

Figure 4: Stress paths in the effective stress plane t',s for two biaxial tests on dense Hostun sand: one drained, the other undrained. 
history can be predicted solely on the basis of the constitutive equations of the solid. The subject of constitutive modeling has received considerable attention in the second part of the last century, especially in geomaterials; the basic concepts of Plasticity have given rise to a large number of variations, including non-normality, multi mechanisms, bounding surfaces, multilaminate models and other Elasto-Plastic extensions of the original framework; on the other hand, Hypoplasticity has been introduced and developed in Europe (Chambon 1989; Kolymbas 1991; Chambon et al. 1994b) from a different point of view, not using the concepts of yield surface and plastic potential -see (Tamagnini, Viggiani, and Chambon 2000) for a synthesis of hypoplasticity at the end of the 90's. Whatever the framework is, the evolution of constitutive equations toward a proper accounting of material behaviour complexity has led to incrementally non-linear constitutive equations (or equivalently rate-type non-linear constitutive equations). These developments have progressed from only two constitutive zones (loading/unloading with respect to a single mechanism), then to several constitutive cones in the stress space (related for example with several plastic mechanisms), and finally to thoroughly non-linear equations, for which any change in strain rate direction induces a change in the tangent constitutive response. As far as the localization criterion is concerned, incremental non-linearity introduces more complexity in the problem. Rice and Rudnicki (Rice and Rudnicki 1980) have discussed in detail the case of mono-mechanism elastoplastic constitutive equations, introducing so-called continuous and discontinuous bifurcation for respectively loading in both inner and outer shear band, and loading inside/unloading outside situations. The framework of the linear comparison solid defined by Hill (Hill 1958) and extended by Raniecki and Bruhns (Raniecki and Bruhns 1981) to non-associated solids makes it possible to establish some theoretical results on the effect of the linearized approach (loading everywhere, i.e. continuous bifurcation) on the localization criterion. In the case of thoroughly non-linear constitutive equations, in general, the localization criterion becomes fully non-linear, which makes necessary some additional assumptions -e.g. Kolymbas in (Kolymbas 1981) - and/or numerical search for solutions. However, in the case of hypoplastic constitutive equations, it has been shown by Chambon and co-workers (Desrues and Chambon 1989; Chambon, Crochepeyre, and Desrues 2000) that due to the mathematical structure of the models, an explicit localization criterion can be derived analytically despite the thoroughly incremental non-linearity of the equations.

It follows from the theoretical studies briefly summarized above, that for most constitutive models for geomaterials a localization criterion can be checked and will predict possible localization on the basis of the pre-bifurcation material characteristics of the material. In its simplest form (incrementally linear rate type law, or linear comparison solid, with tangent constitutive tensor $L$ ), the bifurcation criterion reads $\operatorname{det}(n L n)=0$ and it is known as the criterion of the acoustic tensor. Such a criterion can be used to predict the onset of localization in finite element calculations.

However, using the bifurcation criterion in the context of practical studies still poses questions:

- First it is well known that mesh-dependency affects strongly the development of shear bands in numerical simulations; this can be overcome by different numerical strategies, among which taking into account enriched continuum media. This is out of the scope of the present paper. See in the same book the paper (Chambon and Collin 2004).

- Then, is the criterion still relevant in the context of hydro-mechanical coupled analysis ?

The second question has been addressed by a number authors since the years 90's , e.g. (Loret and Prevost 1991) on a theoretical basis : these authors stated that "the existence of a stationary discontinuity is only dependent upon the material properties of the underlying drained porous solid skeleton". The same conclusion can be deduced from the experimental results summarized above, illustrated by figure 4. On the other hand, (Benallal and Comi 2003) find the same result for associative flow rule for the skeleton, but for non-associative flow rule they find that "unbounded growth occurs when either the condition of localization under drained or undrained conditions is first passed". Numerical modelling of saturated porous media with prediction of strain localization is reported by different authors, e.g. (Schrefler, Sanavia, and Majorana 1996; Ehlers and Volf 1999) and others -see in this book (Chambon and Collin 2004) for a review.

In the present state of affairs, when attempting to model complex problems like e.g. those posed by the assessment of safety of nuclear waste disposal in argiliceous rock layers, it is worth checking if the observed behaviour of totally or partially undrained specimens can be properly captured 


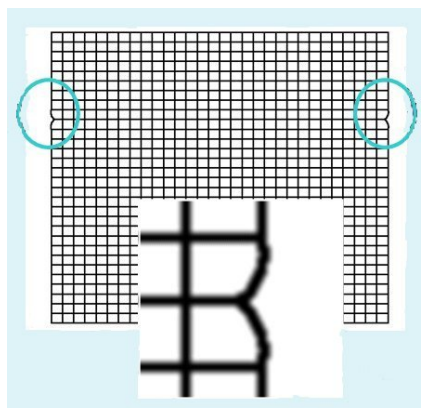

(a) Geometry

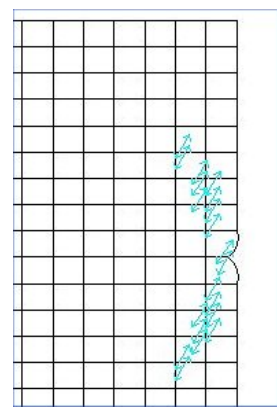

(b) Bifurcated elements at $2.185 \%$

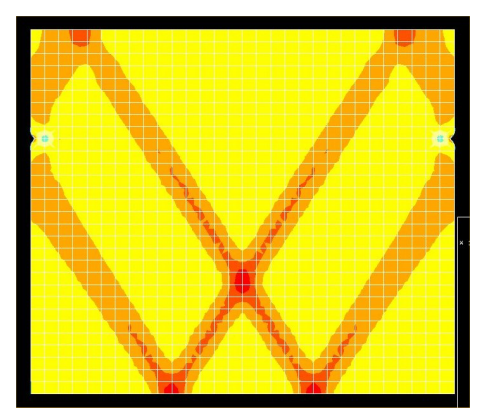

(c) Strain field at $2.5 \%$

Figure 5: Imperfect specimen : localization prediction.

by the hydro-mechanical coupled finite element formulation used, up to the onset of strain localization. In the sequel we present a set of numerical simulations performed to study the response of CLoE constitutive model, equipped with its fully non-linear bifurcation criterion, implemented in the large strain finite element code Lagamine, in hydro-mechanical coupled simulations of totally and partially undrained biaxial experiments.

\section{NUMERICAL SIMULATION OF UNDRAINED AND PARTIALLY DRAINED BIAXIAL EXPERIMENTS}

\subsection{FEM code and constitutive law used}

FEM code Lagamine is developed at Liège University since early seventies. This is a large strain FE code, including a large number of constituve laws related to geomaterials (Elasto-plastic, Hypoplastic, Elasto-visco-plastic). A full Newton-Raphson method is used in the iterative procedure to solve highly non-linear applications. For the hydromechanical problem described in the following, an implicit scheme and a monolithic approach are used; all the coupling terms of the stiffness matrix are evaluated at each iteration. For an extensive description of the Lagamine code and coupling formulation, the reader should refer to (Charlier 1987) or (Collin 2003).

Constitutive model CLoE is developed in Laboratoire $3 S$ in the University of Grenoble, France. It is a Hypoplastic model, whose main characteristics are to be an incrementally thoroughly nonlinear model, not to make use of a decomposition of the strain rate into a elastic and a plastic part, and to allow to derive an analytical bifurcation criterion for the onset of shear banding, despite the strong non-linearity of the model. As discussed in (Desrues and Chambon 2002), the shear moduli used in the model can calibrated using the experimental observations of shear bands in laboratory tests. The basics of the model, and some comparative information with other models can be found in (Chambon, Desrues, Charlier, and Hammad 1994b; Tamagnini, Viggiani, and Chambon 2000; Tamagnini, Viggiani, and Chambon 2001).

A set of numerical experiments were performed on the case of plane strain biaxial compression tests on water-saturated, parallelepipedic specimens. The material is a cohesionless granular medium. The geometry of the specimen is either a perfect parallelepiped, or a slightly imperfect one, the imperfection being a small notch on the side of the specimen (see figure 5(a)). As far as drainage is concerned, the boundaries of the specimen are impervious everywhere in the globally undrained tests, but in drained tests only the four lateral face (vertical) are impervious while water can flow through the top and bottom horizontal faces. The material parameters used are representative of the Hostun RF-S28 sand, dense (void ratio 0.666). The fluid parameters are representative of water, with a density of $1000 \mathrm{~kg} / \mathrm{m}^{3}$, a compressibility of $0.3310^{-12} \mathrm{~Pa}^{-1}$, the viscosity is $10^{-3}$ Pa.s and the intrinsic permeability of the solid is $3.4210^{-11} \mathrm{~m}^{2}$. The initial stress state and the loading program is the same in every case (except the loading rate, see later): initial pore pressure $100 \mathrm{kPa}$, initial overall pressure $200 \mathrm{kPa}$, plane strain in the plane parallel to the front face, constant normal stress on the two lateral vertical faces, axial compression at constant rate 


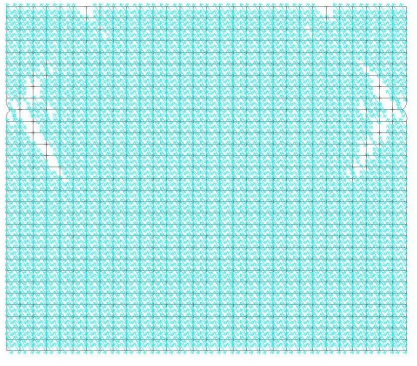

(a) Bifurcated elements

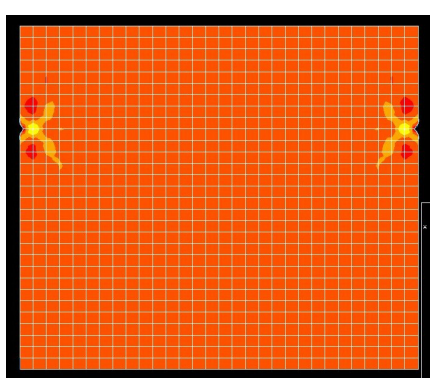

(b) Strain field

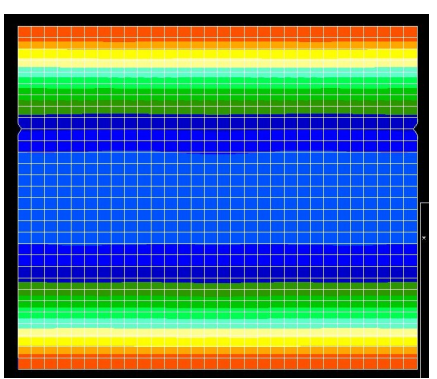

(c) Pore pressure field

Figure 6: Fast open-drainage test at 2.5\%.

(displacement controlled).

The features that we are looking at in the response and in its evolution are :

i) localization prediction by the local bifurcation criterion : first detection, distribution in the specimen; ii) spontaneous localization of the deformation in the numerical specimen; iii) overall force-displacement response.

In order to explore the different possible drainage conditions evocated in section 2.1 , we consider the following cases :

i) dry material; ii) saturated material, open drainage (permeable top and bottom faces): slow / fast / very fast; iii) saturated material, closed drainage (impervious top and bottom faces): slow / fast / very fast; iv) saturated material, delayed drainage (very fast loading, then constant load while drainage takes place). When relevant, the comparison between perfect and imperfect specimen geometry is considered.

\section{RESULTS}

\subsection{Perfect-imperfect geometry}

In the ideal case, dry material and perfect geometry, the bifurcation criterion is met simultaneously in all the elements (more precisely in all the Gauss integration points) at a global axial strain in the interval $[2.23 \%, 2.30 \%]$, with an orientation angle of 60.74 degrees between the normal vector $\vec{n}$ to the shear band and the major principal stress direction -which is vertical everywhere in this perfect case. For comparison, performing local integration of CLoE law along the same loading path with a small integration pace we get localization at $2.244 \%$ with an orientation angle of 60.46 degrees, which is perfectly consistent. Further loading does not produce spontaneous localization in the specimen, but convergence becomes more and more difficult, then impossible.

In the imperfect case (notched specimen), bifurcation is detected in $2 \times 12$ elements (symmetry) in the vicinity of the notch at $\varepsilon_{\text {axial }}=2.185 \%$, then in all elements at $2.37 \%$. Figure 5 (b) shows the spatial organization of the locally bifurcated elements : starting from the notch, the set of bifurcated Gauss points shares in two sub-sets aligned in two beginning shear bands with approximately the same direction as indicated by the criterion itself in each point (only one direction of the two symmetric directions with respect to the local principal stress direction is plotted). Further loading makes the zones of intense shear to propagate; at $\varepsilon_{\text {axial }}=2.5 \%$, deformation is localized in two symmetrical bands with reflection on the rigid boundaries, illustrated by Figure 5(c). As recalled in the introduction, it is well-known that such numerical description of shear banding is strongly mesh-dependent. Again convergence becomes more and more difficult and finally impossible, still later than in the perfect case. As far as the load vs. axial strain curve is concerned, no difference is observed up to $2.5 \%$, when convergence becomes difficult.

What can be concluded is i) the notch triggers a spontaneous numerical localization; ii) in the same time, local bifurcation criterion is met in the zones where numerical localization starts; iii) without imperfections, homogeneous response is maintained (no numerical localization) but convergence becomes impossible sooner, which may be linked to the fact that the bifurcation criterion is met everywhere at the same time in the homogeneous case. 


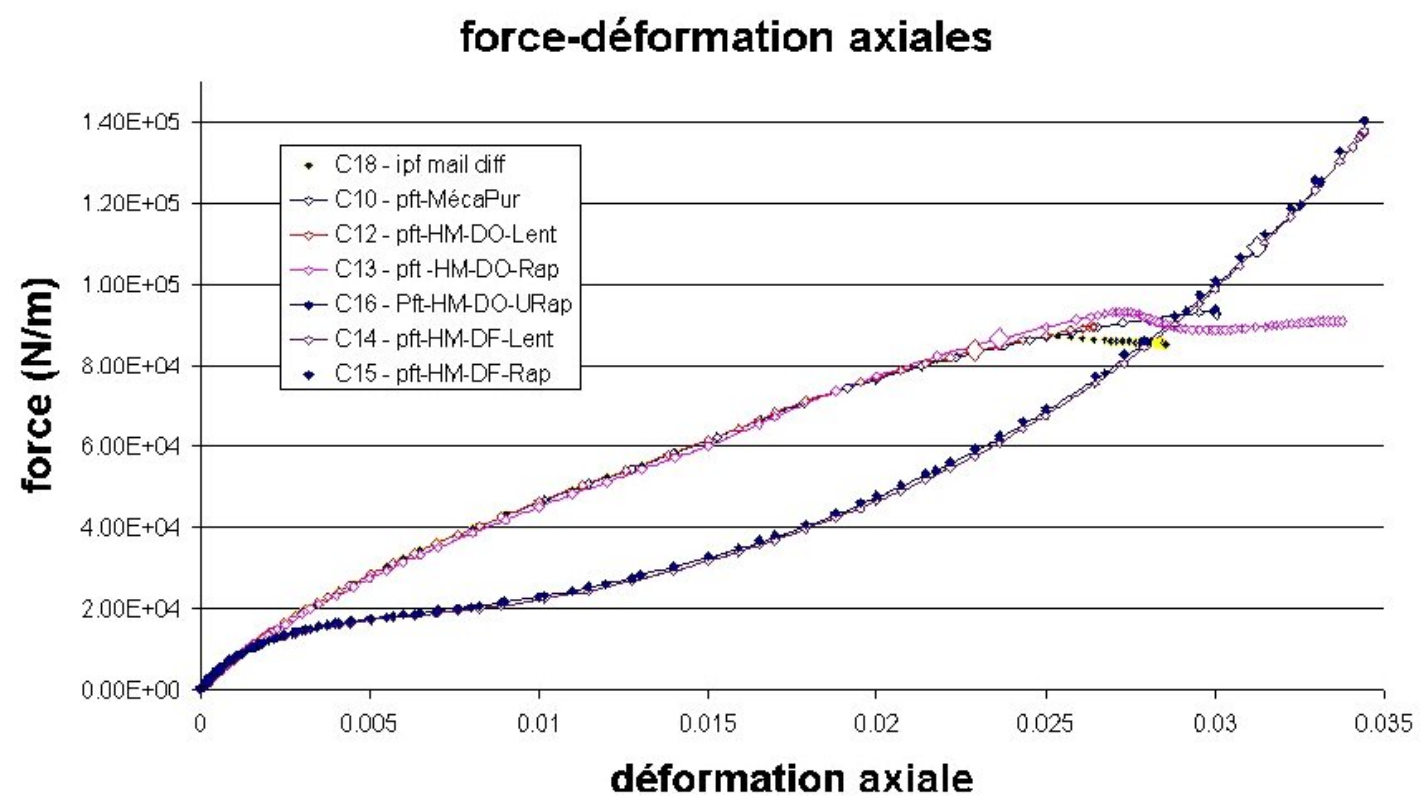

Figure 7: load versus axial-strain comparison : two bundle of curves appear clearly, with respectively : Fast (C13) and slow (C12) coupled simulations giving the same response as the dry case, perfect (C10) and imperfect (C18), while the Very Fast Open-drainage (C16) and the Slow (C14) and fast (C15) Closed-drainage coupled simulations give another, quite different, response.

\subsection{Drained versus undrained loading}

\subsubsection{Dry versus drained loading}

A fully coupled hydromecanical analysis of the imperfect specimen with the same loading program and a small strain rate is compared to the dry analysis. Bifurcation is detected at the same axial deformation, starting in the same few elements then generalizing in the complete specimen. The overall heterogeneity of the deformation is the same as in figure 5(c). As far the pore pressure field is concerned, only limited fluctuations are detected even when the localized shear is well developped. The load-strain curve is identical to the dry imperfect case. These observations were expected, they simply mean that the Hydro-Mechanical coupling implementation does not generate spurious predictions in the perfectly drained limiting case.

\subsubsection{Slow versus Fast loading}

The slow test simulation discussed above was performed at the actual experimental rate, namely $1.2 \mathrm{~mm} / \mathrm{sec}$, which corresponds to an axial strain rate of $0.012 \mathrm{~min}^{-1}$. The fast test is one thousand times faster. In the latter case, bifurcation is detected somewhat later (in $2 \times 4$ elements in the vicinity of the notch at $\varepsilon_{\text {axial }}=2.216 \%$, then in $2 \times 135$ elements at $2.34 \%$, then almost all elements, see Figure 6(a)). The striking difference with the slow (and the dry) cases is that no strain localization appears spontaneously in the specimen : more precisely the beginning of localized shear initiated by the notches does not propagate, as shown by Figure 6(b) which is to compare to Figure 5(c). Accordingly, the pore pressure field is organized with contours essentially parallel to the draining faces, see Figure 6(c), although the gradient is not negligible : maximum pore pressure drop is about $66 k P a$ in the middle of the specimen at $\varepsilon_{\text {axial }}=2.5 \%$, to compare to $0.014 k P a$ in the slow case. In this test we see that $\mathrm{HM}$ coupling is active, drainage is no more perfect and the effective stress inside the specimen is significantly heterogeneous (pore pressure field perturbated by $66 \%$ of the initial homogeneous pore pressure) although quite regular in its organization; indeed, "numerical" strain localization does not take place, although bifurcation in localized mode is detected by the local bifurcation criterion. 


\subsubsection{Very Fast loading}

Up to now, all the simulations performed give almost the same load versus axial strain curve, as shown on figure 7 by the upper bundle of curves. On these curves, the big losange symbol indicate the first detected bifurcation. The major difference between the curves in this bundle is the delayed first bifurcation in the fast test C13, and a slightly stiffer response of the latter after $\varepsilon_{\text {axial }}=2.0 \%$.

Now we consider the very fast loading simulation of the open-drainage test. Again a multiplying factor of 1000 on the strain rate is used with respect to the fast case, so we are $10^{6}$ time faster than the basic drained test.

Like in the fast case, "numerical" localization does not develop. Unlike the fast case however, bifurcation is detected only in a few elements, not located in the vicinity of the notch, but in the immediate vicinity of the drained faces. No special difficulty is encountered with convergence, the simulation continues and the load increases in an accelerated way (see Figure 7, curve C16).

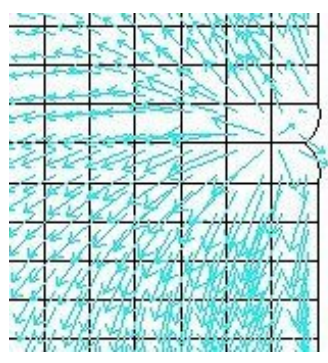

Figure 8: Flux near the notch in slow, globally undrained, simulation (Closed drainage).

\subsubsection{Impervious boundaries : globally undrained case}

With impervious boundaries, slow and fast strain rate simulations gives very close results : no bifurcation detected, no "numerical" localization observed, despite some heterogeneities taking place in the vicinity of the notches (e.g. flux near the notch in Figure 8). As far as the global load versus axial strain response is concerned, Figure 7 shows that the curves $\mathrm{C} 14$ and $\mathrm{C} 15$, respectively relative to the slow and the fast case, are almost coincident with the Open-drainage Very Fast simulation $\mathrm{C} 16$.

\subsection{Delayed drainage}

In order to check if localization, once blocked by the fluid-skeleton interplay, could be released by progressive drainage with time, a simulation of delayed drainage has been performed. In this case, first we perform the same simulation as in the very fast open drainage simulation, up to an axial load much larger than the strength of the drained specimen; then we block the axial deformation and we let the fluid enter the specimen with time (Figure 9(a)). Figure 9(b) shows the evolution of the pore pressure in three locations: near the draining face, at one quarter, and at the middle of the specimen. The first part of the curves is linked to the loading process, with first contractancy (increasing pore pressure), then dilatancy (strong decrease of the pore pressure). Then drainage takes place, and all the curves tend to the imposed back pressure of $100 \mathrm{kPa}$. As far as localization

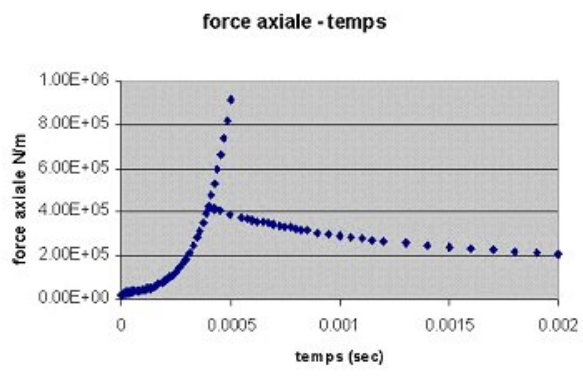

(a) Loading and delayed drainage

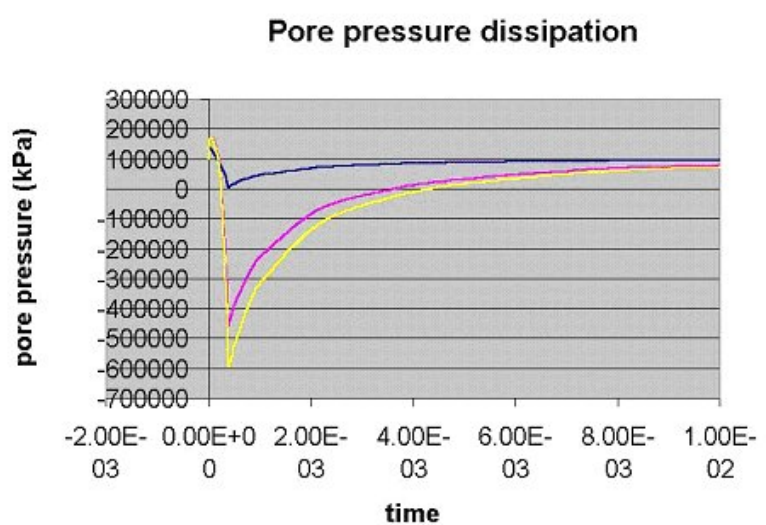

(b) Pore pressure evolution

Figure 9: Delayed Drainage simulation. 


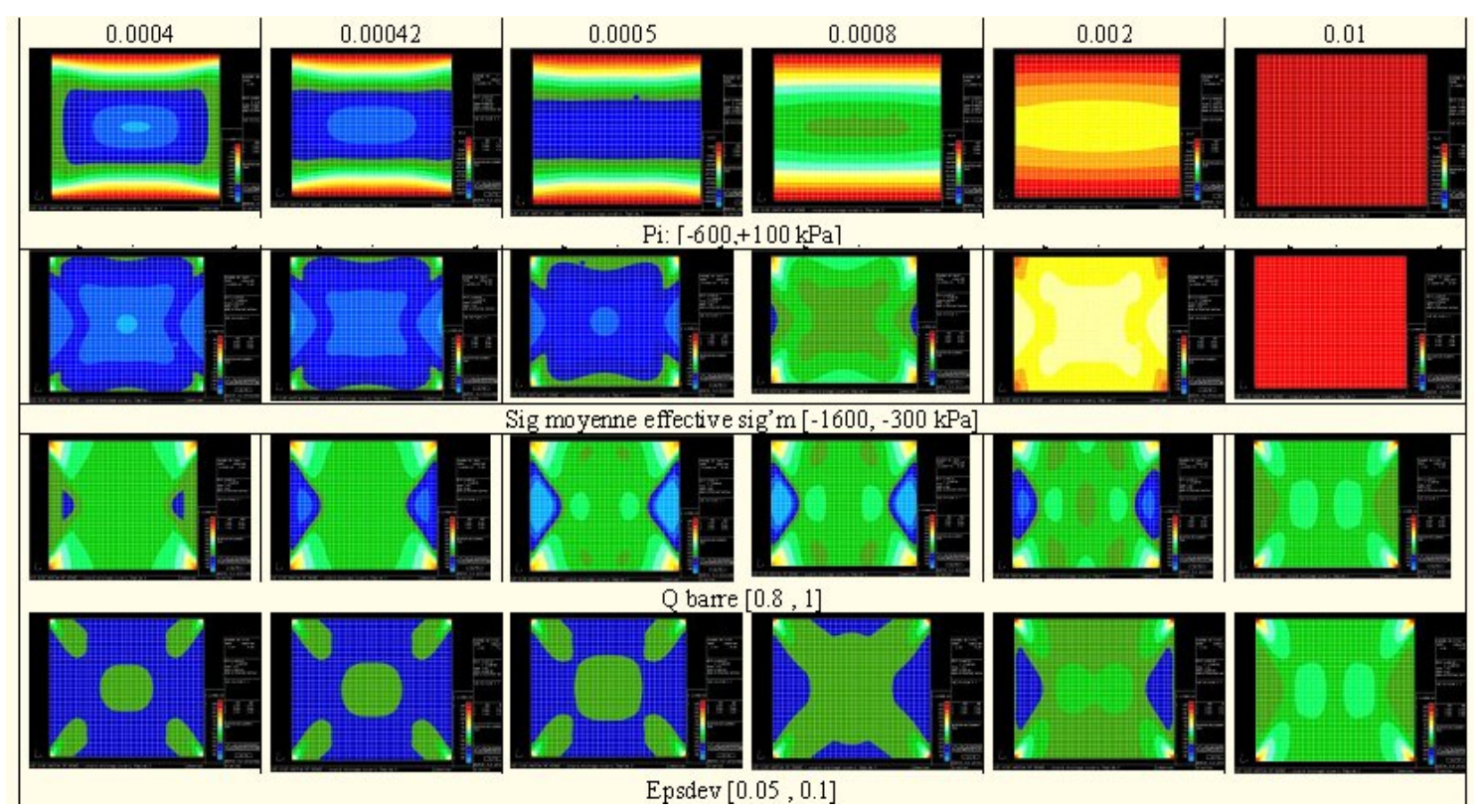

Figure 10: Pore pressure, mean effective stress, mobilized friction, and strain maps during the Delayed Drainage phase.

is concerned, the expected effect is not observed in this simulation: despite the fact that at the time when the loading is stopped, a few elements located at the corners of the specimen were bifurcated, these zones do not extend during the delayed drainage; consistently, no "numerical" localization is observed in the strain maps presented in Figure 10, Bottom line. On the same figure, it can be seen that both the pore pressure (top line) and the mean effective stress (second line) homegeneise with time. The third line presents a quantity which can be described as the local mobilized strength; essentially it shows that this indicator changes somewhat in distribution during the drainage, but does not increase significantly in a localized zone.

\section{CONCLUSIONS}

The implementation of Hydro-Mechanical coupling in the FEM code Lagamine allows to reproduce the main features observed experimentally with respect to strain localization in drained, undrained and partially undrained specimens subjected to biaxial tests in the laboratory. In particular, the resistance to localization induced by pore pressure drop in dilatant specimens is properly modelled. Complex situations, like delayed drainage, can be studied numerically, and parametric studies can be performed to analyse and to design experimental programs.

Acknowledgement Thanks are due to Robert Charlier, from University of Liège, and the Belgian Foundation for scientific research FNRS, for having invited the first author for a stay in Liège, during which this work was performed in close collaboration with the second author.

\section{REFERENCES}

Arthur, J. and T. Dunstan (1982). Rupture layers in granular media. In P. Vermeer and H. Luger (Eds.), IUTAM Conf. Def. Fail. Gran. Media, pp. 453-9. Balkema.

Arthur, J., T. Dunstan, Q. Al-ani, and A. Assadi (1977). Plastic deformation and failure in granular media. Géotechnique 27, 53-74.

Benallal, A. and C. Comi (2003). Perturbation growth and localization in fluid-saturated inelastic porous media under quasi-static loadings. Journal of the Mechanics and Physics of Solids 51, 851-899.

Bésuelle, P., J. Desrues, and S. Raynaud (2000). Experimental characterisation of the localisation phenomenon inside a vosges sandstone in a triaxial cell. Int. J. Rock Mech. \& Mining Sci. 37, 1223-37. 
Chambon, R. (1989). Une classe de lois de comportement incrémentalement nonlinéaires pour les sols non visqueux - résolution de quelques problèmes de cohérence. C. R. Acad Sci. Paris 308, 1571-1576.

Chambon, R., D. Caillerie, J. Desrues, and S. Crochepeyre (1999). A comparison of incremental behavior of elastoplastic and cloe models. International Journal for Numerical and Analytical Methods in Geomechanics 23, 295-316.

Chambon, R. and F. Collin (2004). Proc. CDM2004, Chapter Hydro-Mechanical numerical modelling of geotechnical problems using local second gradient models.

Chambon, R., S. Crochepeyre, and J. Desrues (2000). Localization criteria for non linear constitutive equations of geomaterials. Mechanics of Cohesive-Frictional Materials 5, 561-582.

Chambon, R., J. Desrues, R. Charlier, and W. Hammad (1994a). Cloe, a new rate type constitutive model for geomaterials: Theoretical basis and implementation. Int. J. Num. Anal. Meth. Geom. 18(4), 253-278.

Chambon, R., J. Desrues, R. Charlier, and W. Hammad (1994b). Cloe, a new rate type constitutive model for geomaterials: Theoretical basis and implementation. Int. J. Num. Anal. Meth. Geom. 18(4), 253-278.

Charlier, R. (1987). Approche unifiée de quelques problèmes non linéaires de mécanique des lilieux continus par la méthode des éléments finis. thèse de doctorat.

Collin, F. (2003). Couplages thermo-hydro-mécaniques dans les sols et les roches tendres partiellement saturés. Thèse de doctorat.

Desrues, J. (1984). La localisation de la déformation dans les matériaux granulaires. thèse de Doctorat es Sciences.

Desrues, J. (1990). Geomaterials constitutive equations and modelling, Chapter Shear Band Initiation in Granular Materials: Experimentation and Theory, pp. 283-310. Elsevier.

Desrues, J. (1998). Localization Patterns in ductile and brittle geomaterials, pp. 137-158. Wiley-Interscience-Europe.

Desrues, J. (2004). Tracking strain localization in geomaterials using computerized tomography. In J. Otani and Y. Obara (Eds.), Proc. of the International Workshop on X-ray CT for Geomaterials, International Workshop on X-ray CT for Geomaterials GeoX 2003, Kumamoto, Japan, November 6-7, 2003, pp. 15-41. Balkema.

Desrues, J. and R. Chambon (1989). Shear band analysis for granular materials: the question of incremental non linearity. Ingenieur Archiv 59, 187-196.

Desrues, J. and R. Chambon (2002). Shear bands analysis and shear moduli calibration. Int. Journal Solids and Structures 39(13-14), 3757-3776.

Desrues, J., R. Chambon, M. Mokni, and F. Mazerolle (1996). Void ratio evolution inside shear bands in triaxial sand specimens studied by computed tomography. Géotechnique 46(3), 529-546.

Desrues, J., J. Lanier, and P. Stutz (1985). Localization of the deformation in tests on sand sample. Engineering fracture mechanics 21, 909-921.

Desrues, J. and G. Viggiani (2004). Strain localization in sand: an overview of the experimental results obtained in grenoble using stereophotogrammetry. Int. Journal for Numerical and Analytical methods in Geomechanics 28(4), 279-321.

Ehlers, W. and W. Volf (1999). Localization phenomena in liquid-saturated and empty porous solids. Transport in Porous Media : 159Ú177, 1999. 34, 159-177.

Finno, R., W. Harris, M. Mooney, and G. Viggiani (1996). Strain localization and undrained steady state of sands. Journal of Geotechnical Engineering, ASCE 122(6), 462-473.

Finno, R., W. Harris, M. Mooney, and G. Viggiani (1997). Shear bands in plane strain compression of loose sand. Géotechnique 47(1), 149-165. 
Han, C. and I. Vardoulakis (1991). Plane-strain compression experiments on water-saturated fine-grained sand. Géotechnique 41(1), 49-78.

Hill, R. (1958). A general theory of uniqueness and stability in elastoplastic solids. JMPS 6, 236-49.

Kolymbas, D. (1981). Bifurcation analysis for samples with a non-linear constitutive equation. Ingenieur-Archiv 50, 131-140.

Kolymbas, D. (1991). An outline of hypoplasticity. Archive of applied mechanics 61, 143-151.

Loret, B. and J. Prevost (1991). Dynamic strain localization in fluid-saturated porous media. Journal of Engineering Mechanics 117(4), 907-922.

Mokni, M. and J. Desrues (1999). Strain localisation measurements in undrained plane-strain biaxial tests on hostun rf sand. Mechanics of cohesive-frictional materials 4, 419-441.

Raniecki, B. and O. Bruhns (1981). Bounds to bifurcation stresses in solids with non associated flow rules at finite strain. JMPS 29, 153-72.

Rice, J. (1976). Theoretical and Applied Mechanics, Chapter The Localization of Plastic Deformation, pp. 207-220. North-Holland Publishing Company.

Rice, J. and J. Rudnicki (1980). A note on some features of the theory of the localization of deformation. Int. J. Solids Struct. 16, 597-605.

Roger, V., J. Desrues, and G. Viggiani (1998). Experiments on strain localisation in dense sand under isochoric conditions. In O. F. (Ed.), Localisation and Bifurcation Theory for Soils and Rocks, 4th Workshop on Localization and Bifurcation Theory for Soils and Rocks, Gifu (Japan) 28 septembre-2 octobre 1997, pp. 239-248. Balkema.

Rudnicki, J. and J. Rice (1975). Conditions for the localisation of deformation in pressure sensitive dilatant materials. JMPS 23, 371-394.

Schrefler, B., L. Sanavia, and C. Majorana (1996). A multiphase medium model for localization and post localisation simulation in geomaterials. Mech. Of Cohes.-Frict. Mat. 1, 95-114.

Tamagnini, C., G. Viggiani, and R. Chambon (2000). A review of two different approaches to hypoplasticity, pp. 107-145. Springer.

Tamagnini, C., G. Viggiani, and R. Chambon (2001). Some remarks on shear band analysis in hypoplasticity, pp. 85-93. Balkema.

Tatsuoka, F., T. Nakamura, C. Huang, and K. Tani (1990). Strenght anisotropy and shear band direction in plane strain test of sand. Soils and Foundations 30(1), 35-54.

Tatsuoka, F., M. Sakamoto, T. Kawamura, and S. Fukushima (1986). Strenght and deformation characteristics of sand in plane strain compression at extremely low pressures. Soils and Foundations 26(1), 65-84.

Vardoulakis, I. (1996a). Deformation of water-saturated sand: I. uniform undrained deformation and shear banding. Géotechnique 46(3), 441-456.

Vardoulakis, I. (1996b). Deformation of water-saturated sand: Ii. effect of pore water flow and shear banding. Géotechnique 46(3), 457-472.

Vardoulakis, I., M. Goldscheider, and Q. Gudehus (1978). Formation of shear bands in sand bodies as a bifurcation problem. Int. J. Num. Anal. Meth. Geom. 2, 99-128.

Vardoulakis, I. and B. Graf (1985). Calibration of constitutive models for granular materials using data from biaxial experiments. Géotechnique 35(3), 299-317. 